${ }^{a}$ Division of Reproductive Health, Clinical Science Research Laboratories, Warwick Medical School; and ' Warwick Systems Biology Centre, University of Warwick, Coventry, England, United Kingdom; 'Interdisciplinary Research Groups of BioSystems and Micromechanics, and Infectious Diseases, SingaporeMIT Alliance for Research and Technology, Singapore, Singapore; dDepartment of Reproductive Medicine, KK Women's and Children's Hospital, Singapore, Singapore; ${ }^{\mathrm{e}}$ Department of Obstetrics and Gynaecology, Juntendo University Faculty of Medicine, Tokyo, Japan; 'Department of Surgery and Cancer, Imperial College London, Imperial Centre for Translational and Experimental Medicine (ICTEM), London, United Kingdom

Correspondence: Jan Brosens M.D., Ph.D. Division of Reproductive Health, Clinical Science Research Laboratories, Warwick Medical School, University of Warwick, Coventry CV2 2DX, United Kingdom. Telephone: +44-24769 68704; Fax: + 44-2476968653; email: J.J.Brosens@warwick.ac.uk

Received June 8, 2015; accepted for publication September 4 , 2015; first published online in Stem Cells Express September 29, 2015.

(c) AlphaMed Press 1066-5099/2015/\$30.00/0

http://dx.doi.org/

10.1002/stem.2222

This is an open access article under the terms of the Creative Commons Attribution-NonCommercial-NoDerivs License, which permits use and distribution in any medium, provided the original work is properly cited, the use is non-commercial and no modifications or adaptations are made.

\title{
Loss of Endometrial Plasticity in Recurrent Pregnancy Loss
}

\author{
Emma S. Lucas, ${ }^{a}$ Nigel P. Dyer, ${ }^{b}$ Keisuke Murakami, ${ }^{a}$ Yie Hou lee, ${ }^{\text {c }}$ Yi-Wah Chan, \\ Giulia Grimaldi, ${ }^{a}$ Joanne Muter, ${ }^{a}$ Paul J. Brighton, ${ }^{a}$ Jonathan D. Moore, ${ }^{b}$ \\ Gnyaneshwari Patel, ${ }^{a}$ Jerry K.Y. Chan, ${ }^{d}$ Satoru Takeda, ${ }^{e}$ Eric W-F. Lam, ${ }^{f}$ \\ SIOBHAN Quenby, ${ }^{a}$ SASCHA OTt, ${ }^{b}$ JAN J. BROSENs ${ }^{a}$
}

Key Words. Epigenetics - Stem cells • Endometrium • Pregnancy • Miscarriage • Decidualization • Senescence $\cdot$ High mobility group protein 2

\section{ABSTRACT}

Menstruation drives cyclic activation of endometrial progenitor cells, tissue regeneration, and maturation of stromal cells, which differentiate into specialized decidual cells prior to and during pregnancy. Aberrant responsiveness of human endometrial stromal cells (HESCs) to deciduogenic cues is strongly associated with recurrent pregnancy loss (RPL), suggesting a defect in cellular maturation. MeDIP-seq analysis of HESCs did not reveal gross perturbations in CpG methylation in RPL cultures, although quantitative differences were observed in or near genes that are frequently deregulated in vivo. However, RPL was associated with a marked reduction in methylation of defined CA-rich motifs located throughout the genome but enriched near telomeres. Non-CpG methylation is a hallmark of cellular multipotency. Congruently, we demonstrate that RPL is associated with a deficiency in endometrial clonogenic cell populations. Loss of epigenetic stemness features also correlated with intragenic CpG hypomethylation and reduced expression of HMGB2, coding high mobility group protein 2 . We show that knockdown of this sequence-independent chromatin protein in HESCs promotes senescence and impairs decidualization, exemplified by blunted time-dependent secretome changes. Our findings indicate that stem cell deficiency and accelerated stromal senescence limit the differentiation capacity of the endometrium and predispose for pregnancy failure. STEM CELLS 2016;34:346-356

\section{Significance Statement}

Recurrent pregnancy loss (RPL) is a common and distressing disorder. While many risk factors have been invoked to explain RPL, the underlying pathological pathways are not understood. We demonstrate that RPL is strongly associated with uterine stem cell deficiency and enhanced cellular senescence. This in turn perturbs endometrial preparation for pregnancy, a process termed decidualization, and persistence of these defects over several conception cycles will lead to consecutive miscarriages. Our findings open up new avenues to screen women prior to pregnancy for the risk of miscarriage and point to the potential of cell-based therapies in the prevention of RPL.

\section{INTRODUCTION}

Between $15 \%$ and $25 \%$ of pregnancies end in miscarriage, defined as the loss of pregnancy before the fetus reaches viability [1]. Most miscarriages are sporadic and occur before 12 weeks gestation. They frequently involve numeric chromosome errors in the conceptus. Recurrent pregnancy loss (RPL), defined by consecutive miscarriages, is viewed as a distinct disorder [2]. It is estimated that $5 \%$ of women experience two clinical miscarriages and approximately $1 \%$ three or more losses [3]. In RPL, the incidence of euploidic fetal loss increases with each additional miscarriage, whereas the likelihood of a future successful pregnancy gradually decreases [4, 5]. Clinically, $\mathrm{RPL}$ is a debilitating disorder, associated with considerable psychological morbidity, for which there is no effective medical intervention. Fortunately, the cumulative live birth rate for most RPL patients is high $[1,4,5]$, which in turn suggests that embryo-endometrial interactions are intrinsically dynamic and capable of adapting to ensure reproductive success [6].

Human embryos are exceptionally diverse in their chromosomal make-up and developmental potential $[7,8]$. Recent studies have 
shown that decidualizing (differentiating) endometrial stromal cells serve as biosensors of embryos that have breached the luminal epithelium $[9,10]$. This observation led to the concept of "natural selection," meaning that decidualizing endometrium responds to embryonic signals in a manner that either supports further development or leads to rapid demise through menstruation-like shedding; thus increasing the likelihood of future reproductive success by safeguarding resources and expediting recovery. Emerging evidence indicates that abnormal decidualization causes loss of the selectivity checkpoint, which renders the endometrium excessively permissive to implantation but unable to sustain the conceptus $[11,12]$. This hypothesis is supported by in vitro studies demonstrating that RPL is associated with aberrant responsiveness of human endometrial stromal cells (HESCs) to deciduogenic signals, although the underlying mechanisms are unknown [13-15].

Decidual transformation of the human endometrium is not dependent on embryo implantation. Instead, this process is initiated during the mid-luteal phase of each cycle in response to the postovulatory rise in progesterone and increasing endometrial cAMP levels [11]. Consequently, decidualization is a reiterative process directly linked to cyclic activation of mesenchymal stem cells (MSCs) and subsequent differentiation into mature stromal cells in regenerating endometrium. We hypothesized that defects in cyclic regeneration of the endometrium in RPL patients impact on the DNA methylation status of HESCs, thus accounting for the aberrant decidual response as well as the functional memory of the cells in culture. Based on an unbiased analysis of the DNA methylome of primary HESC cultures, we demonstrate that the endometrium associated with RPL exhibits loss of plasticity, defined by stem cell deficiency, heightened senescence, and limited differentiation potential.

\section{MATERIALS AND MetHods}

\section{Patient Selection and Endometrial Sampling}

The study was approved by the NHS National Research Ethics-Hammersmith and Queen Charlotte's \& Chelsea Research Ethics Committee (1997/5065). For the MeDIP-seq analysis, eight endometrial biopsies were obtained randomly in the cycle. All other biopsies were timed between 6 and 10 days after the preovulatory luteinizing hormone surge. None of the subjects were on hormonal treatments for at least 3 months prior to the procedure. Written informed consent was obtained from all participants in accordance with the guidelines in The Declaration of Helsinki 2000. A total of 183 endometrial biopsies were used in this study. Demographic details are summarized in Supporting Information Table S1.

\section{Primary Cultures}

HESCs were isolated from endometrial tissues as described previously [16]. Purified HESCs were expanded in maintenance medium of Dulbecco's modified Eagle's medium (DMEM)/F-12 containing $10 \%$ dextran-coated charcoal-treated fetal bovine serum (DCC-FBS), L-glutamine (1\%), and $1 \%$ antibioticantimycotic solution. Confluent monolayers were decidualized in DMEM/F-12 containing 2\% DCC-FBS with $0.5 \mathrm{mM}$ 8-bromocAMP (Sigma-Aldrich, Poole, U.K., http://www.sigmaaldrich. com) and $10 \mu \mathrm{M}$ medroxyprogesterone acetate (Sigma-
Aldrich) to induce a differentiated phenotype. Isolation of isolated $\mathrm{W}_{5} \mathrm{C5}^{+}$- and $\mathrm{W} 5 \mathrm{C5}^{-}$-cells and colony-forming assays were performed as described previously [17].

\section{Transfection and RNA Interference}

Primary HESCs were transfected in complete media at approximately $80 \%$ confluence using JetPrime Reagent (Polyplus-transfection SA, Illkirch, France, http://www.polyplus-transfection.com/) and either SMARTpool targeting small interfering RNA (siRNA) (50 nM; GE Healthcare, Little Chalfont, U.K., http://dharmacon.gelifesciences.com/) or nontargeting (NT) control. Culture media was changed after 24 hours. For high mobility group protein 2 (HMGB2) knockdown studies, transfected cells were either maintained undifferentiated or decidualized for 8 days as described above. For DNMT3A knockdown studies, cells were harvested at 24 hours after transfection and subjected to matrix-MeDIP-qPCR analysis.

\section{RNA Sequencing}

For RNA sequencing, RNA was treated with Amplification grade DNase I (Life Technologies, Rockville, MD, http://www. lifetech.com), followed by ethanol precipitation and clean-up according to standard protocols. RNA sequencing was performed by Source Bioscience (Nottingham, U.K., http:// www.lifesciences.sourcebioscience.com/) using TruSeq Stranded mRNA Library Preparation and standard Illumina Protocols for sequencing on HiSeq 2000. RNA integrity number for all samples was $\geq 8.2$. Transcriptomic maps of singleend reads were generated using bowtie-2.2.3, samtools0.1.19, and tophat-2.0.12 against the University of California Santa Cruz (UCSC) hg19 reference transcriptome (2014) from the Illumina iGenomes resource using the fr-firststrand setting. Transcript counts were assessed by HTSeq-0.6.1 using the reverse strand setting and intersection-non-empty mode and counts were assigned to gene IDs. Transcripts per million were calculated as recently described [18]. Count data from the TopHat-HTSeq pipeline were analyzed using three different methods for differential expression detection, that is, DESeq2, baySeq, and edgeR [19-22]. Expression data have been submitted to the GEO repository (GSE65102).

\section{MeDIP-seq and CpG Methylation Analysis}

MeDIP-seq was performed by Arraystar (Rockville, MD www.arraystar.com). Briefly, DNA was fragmented to 100-500 bp fragments by sonication using a Bioruptor (Diagenode, Seraing, Belgium https://www.diagenode.com/). One $\mu$ g DNA was used for end repair and adapter ligation and then immunoprecipitated with an anti-5-methylcytosine antibody (Diagenode). After polymerase chain reaction (PCR) amplification for enrichment of precipitated fragments, 200-300 bp DNA fragments were gel-extracted and quantitated using Agilent 2100 Bioanalyzer (Agilent Technologies, Santa Clara, CA, http:// www.agilent.com). Libraries were denatured and sequenced on the Genome Analyzer IIx following the TruSeq SBS Kit v5 protocol. Image analysis and base calling were performed using Off-Line Basecaller software (OLB V1.8). After passing the Solexa CHASTITY quality filter, clean reads were aligned to the Human genome (UCSC Hg19) using bowtie software (V0.12.7). Approximately, 94 million uniquely mapped reads, representing $>3.3$ billion bases, were obtained for each sample. Each read was extended to $200 \mathrm{bp}$ in length. A 

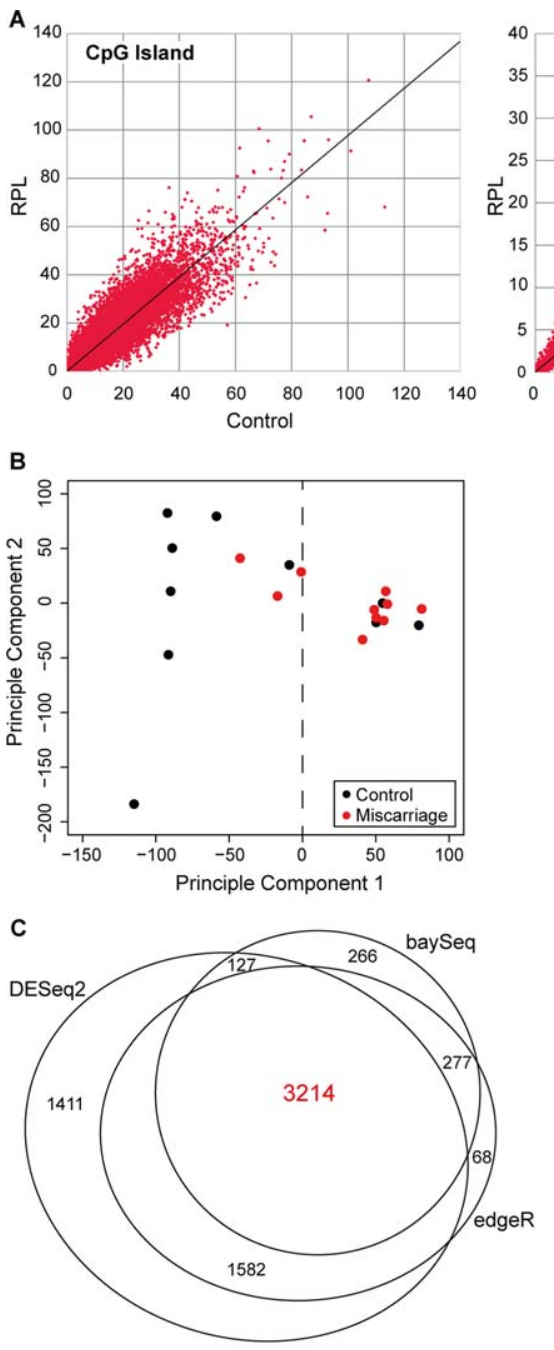

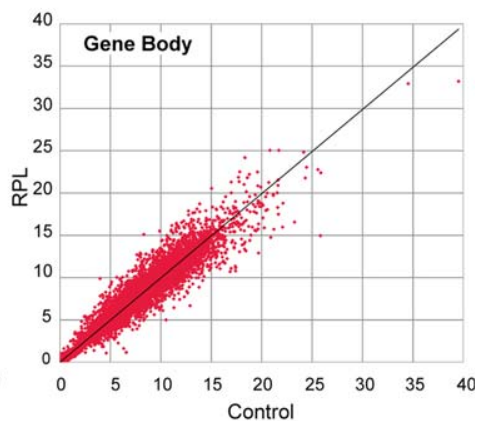

D
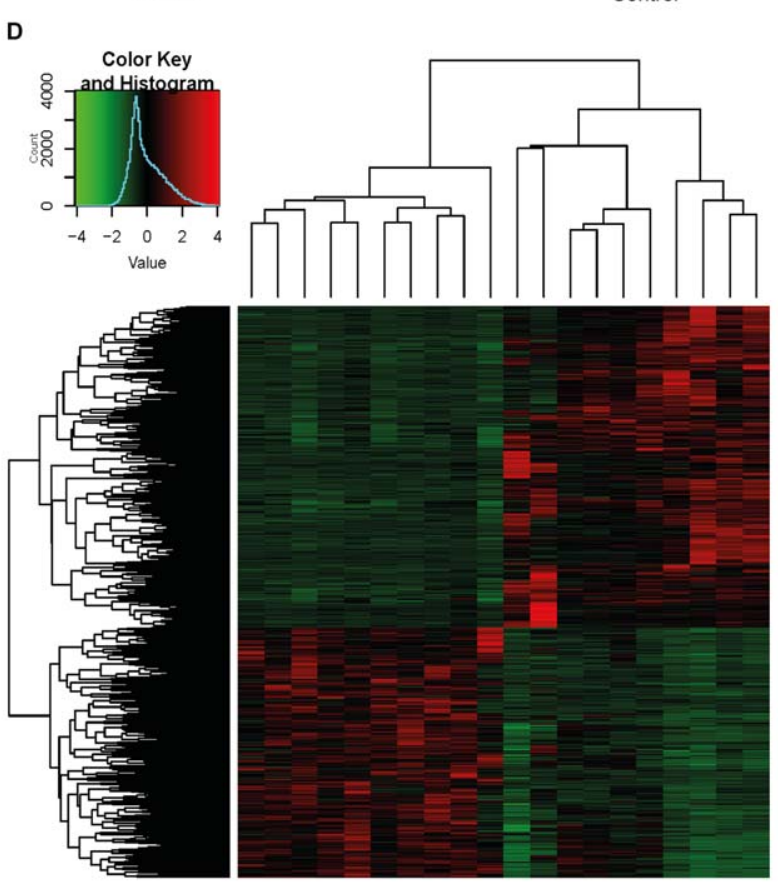

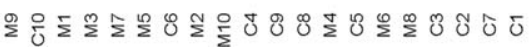

Figure 1. Differential CpG methylation in primary human endometrial stromal cells (HESCs) and altered endometrial gene expression associated with RPL. (A): Comparison of average MeDIP-seq scores, reflecting absolute levels of CpG methylation per kb (read counts per $\mathrm{kb})$, of primary HESC cultures established from RPL patients $(n=4)$ and control subjects $(n=4)$. Overall methylation at CGIs (slope $=0.977$ ), promoter regions (slope $=0.986$ ), and within gene bodies (slope $=0.996$ ) was comparable between the sample sets. (B): Principle component analysis of RNA-seq data from 20 mid-luteal whole endometrial biopsies segregates samples into cluster 1, enriched in non-RPL samples (control, C); and cluster 2, encompassing 7 out of 10 biopsies obtained from miscarriage patients (M). (C): A proportional Venn diagram of differentially expressed transcripts identified by DESeq2, baySeq, and edgeR analyses. (D): Clustered heatmap of top-ranked transcripts differentially expressed between clusters 1 and 2. Abbreviation: RPL, recurrent pregnancy loss.

methylation score for any region in the genome was defined as the number of extended reads per kb [23]. The DNA methylation status of a specific region (e.g., promoter or gene body) was defined as unmethylated, partially, or fully methylated if the MeDIP score was $<4.25$ reads per $k b$, between 4.25 and 18.28 reads per $k b$, or $>18.28$ reads per $k b$, respectively. The MeDIP-seq data were deposited to the GEO repository (GSE65102).

\section{MeDIP-seq Peak Characteristics and Scatter Plots}

Gene body locations are as defined in the UCSC hg18-based definitions provided as part of CisGenome. Gene promoters were taken as the first $500 \mathrm{bp}$ upstream of the gene start position. CA/TG, Alu, and L-1 regions are as annotated in the UCSC hg18 genome data. All regions less than $100 \mathrm{nt}$ in length were ignored as were all regions with fewer than 20 fragments in any of the samples used. Fragments were counted if they overlapped the annotated region. The counts are normalized to compensate for differences in total counts between each of the samples and also the variation in region length. Overall methylation values for a sample group (patients, controls) are the geometric mean of the values from each of the samples.

\section{Analysis of Hypomethylation of CA-Rich Regions}

Initial examination of the MeDIPseq data showed sample 6 to be an outlier in terms of methylation levels in CA-rich regions. Consequently, control sample methylation was calculated from samples 1-4 and RPL methylation from samples 5, 7, and 8 . Figure $2 \mathrm{~B}$ shows that a subset of annotated CA-rich regions was hypomethylated, with most of such regions exceeding a fold-change threshold of 1.5 (with a 1.5 foldchange dividing the region between red and grey lines in Fig. 2B into equal halves). To expand analyses to all such regions 
A

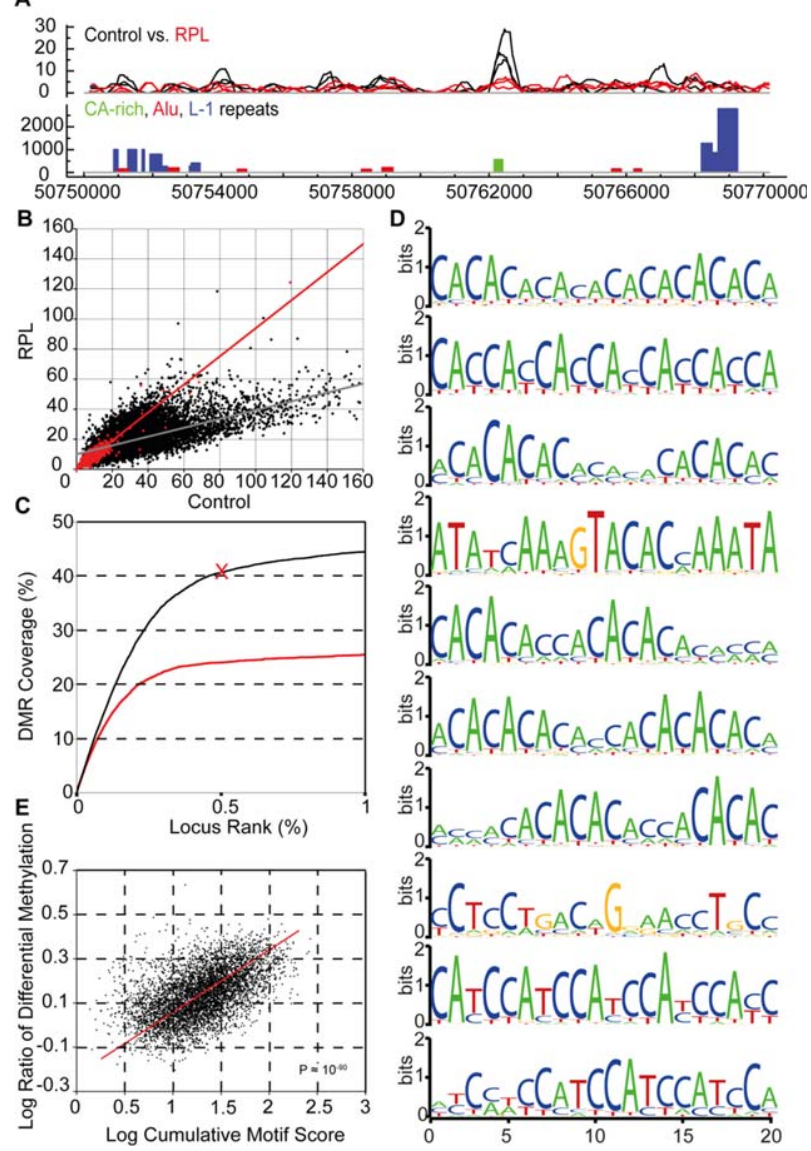

Figure 2. RPL is associated with loss of methylation at distinct CA-rich genomic loci. (A): Example of differential methylation between control and RPL cultures (top panel) at a CA-rich region on chromosome 6. A divergent pattern of methylation was not observed at other repetitive genomic elements (bottom panel). Loss of methylation at CA repeats was observed in all but one RPL culture (biopsy 6, Table E1), which was excluded from further analyses. (B): Average methylation at annotated CA-rich loci (black dots) in control versus RPL cultures. For comparison, CpG methylation in gene bodies (red dots) is also shown. (C): Genomic sequences identified based on motifs are more predictive for differential methylation than CA-richness alone. $x$-axis shows the rank $n$ of loci when sorted by motif-model score (black curve) or CA-richness (red curve). $y$-Axis shows the fraction of DMRs included in the top $\mathrm{n}$ loci. For example, the red cross indicates that $41 \%$ of DMRs are included in the top $0.5 \%$ of the regions detected by the motif model, while the remaining $59 \%$ of the DMRs are in the other $99.5 \%$ of the genome. (D): Top 10 motifs predictive of differentially methylated CA-rich regions. (E): The motif model can meaningfully predict the extent of differential methylation. $x$-Axis shows the predicted methylation ratio and $y$ axis shows the measured methylation ratio. Each DMR is represented by a single dot. Abbreviations: DMR, differentially methylated region; RPL, recurrent pregnancy loss.

in the genome, we scanned the genome for all loci that showing $50 \%$ or more reduction in methylation in RPL compared to control subjects and used this set of 2741 differentially methylated regions (DMRs) in subsequent analyses.

\section{Motif Modeling of CA-Rich DMRs}

Differentially methylated peaks were ordered by methylation ratio, defined as the ratio of methylation scores between control and RPL (with exclusion of sample 6), without application of a count or length threshold but with addition of a pseudo count of 10 to all of the densities prior to ratio calculation to avoid assigning significance to peaks where the total fragment counts are low. The Gibbs Motif Mapper, implemented in CisGenome [24], was used to identify over-represented motifs based on the sequences of the top 200 DMRs, searching for 10 motifs of length 20 with a background genome model determined by a Markov model of order 0 , with modification to allow the inclusion of repeat-masked data. CisGenome Motif Mapping was then used to identify those locations in the genome where there was a match between the sequence and each of the motifs, setting the likelihood threshold to 10,000 , including repetitive regions and using a background model estimated from the input sequences with a Markov model of order 0 . The degree to which motif matches were over-represented within the top 2,741 DMRs was found to be between a factor of 7 and 240. DMRs were predicted solely on the basis of the DNA sequence. At each nucleotide location a cumulative log likelihood of all the motif matches that overlap the location was calculated, and a candidate DMR was identified wherever this value exceeded 60 for a minimum length of $250 \mathrm{nt}$, allowing the value to drop below this value for lengths of up to $120 \mathrm{nt}$ within the region. The model parameters were learned using a subset of the data, using the remaining data to confirm that overfitting did not occur. A score equal to the cumulative value across the region is assigned to the region. A total of 2,419 candidate DMRs were found. To compare the motif-based method of DMR prediction with prediction using simple CA/TG repeat annotation a second set of motif-based region predictions were calculated with a lower threshold of 10 and a reduced length threshold of $120 \mathrm{nt}$. The genome was divided into $2 \mathrm{~kb}$ windows and two scores calculated for each window: a cumulative score of all the annotated CA regions within the window and the second using the motif-based DMR prediction scores. The differential methylation score was calculated for each window based on the ratio of the geometric means of the normalized MeDIP fragments for the control and RPL samples.

\section{Matrix-MeDIP}

RNA-free DNA was fragmented using a Bioruptor (Diagnode) over 12 low-power on (30 seconds) and off (90 seconds) cycles. Sonication was performed in Tris-EDTA (TE) buffer, pH 8.0 at a concentration of $2 \mu \mathrm{g}$ DNA in $100 \mu \mathrm{lTE}$. After sonication, DNA was ethanol precipitated and resuspended in $20 \mu \mathrm{l}$ elution buffer ( $1 \%$ SDS and $100 \mathrm{~mm} \mathrm{NaHCO}$ ) and boiled at $100^{\circ} \mathrm{C}$ for 10 minutes. Denatured DNA was chilled on ice and stored at $-80^{\circ} \mathrm{C}$. Matrix-MeDIP (microplate-based methylated DNA immunoprecipitation) was performed as described [16]. Briefly, polystyrene 96-well plates were UV exposed for 48 hours and then coated with protein A $(0.5 \mu \mathrm{g}$ per well; Sigma-Aldrich) in phosphate buffered saline (PBS). Sonicated DNA was immunoprecipitated with anti-5-methylcytosine antibody $(1 \mu \mathrm{g}$ per $\mu \mathrm{g}$ DNA; Cambridge Bioscience, Cambridge, U.K., www.bioscience.co.uk/) at $4^{\circ} \mathrm{C}$ for 1 hour in a sonicating water bath. Mouse IgG1 was used as an isotype control (Cell Signaling Technology, Beverly, MA, http://www.cellsignal.com). During this time, protein A coated wells were washed in PBS and blocked with $5 \%$ bovine serum albumin (BSA) in immunoprecipitation (IP) buffer for 1 hour at room temperature (RT). Blocking solution was aspirated, IP reactions were added to 
wells and the plates then placed on ice for 1 hour. Wells were washed four times in IP buffer and once in TE, $\mathrm{pH} 7.6$, before addition of elution buffer. DNA was eluted by incubation at $55^{\circ} \mathrm{C}$ for 30 minutes and then at $95^{\circ} \mathrm{C} 15$ minutes in a thermal cycler. Eluted DNA was subjected to real-time quantitative PCR. Briefly, reactions were prepared using $2 \times$ Power SYBR master mix (Life Technologies), $300 \mathrm{nM}$ primer, and $2 \mu \mathrm{l}$ eluted DNA in a $20 \mu \mathrm{l}$ final reaction volume. Thermal cycling was performed with 10 min activation at $95^{\circ} \mathrm{C}$ and 45 cycles of $95^{\circ} \mathrm{C}$ for 15 seconds then $60^{\circ} \mathrm{C}$ for 1 minute. Reaction specificity was confirmed by dissociation curve analysis. Primer efficiencies $(E)$ were calculated from 7 log standard curves. Relative enrichment of methylated targets was calculated as $E\left(C t_{\text {input }}-C t_{\text {MeDIP }}\right) \times X$, where $X$ represents the percentage of DNA in the input versus MeDIP wells. For multiplate studies, a calibrator sample (constant) was included on all plates. Enrichment values are expressed normalized to the IgG1 control $(\operatorname{IgG1}=1)$. Real-time PCR examination of known hypermethylated (BRD1) and hypomethylated (COQ3) control regions confirmed sensitive and specific enrichment of methylated DNA by the matrix-MeDIP approach (Supporting Information Fig. S1). Primer sequences are detailed in Supporting Information Table S2.

\section{Western Blotting}

Whole cell protein extracts were prepared from transfected cells in RIPA buffer containing protease inhibitors (complete Mini; Roche Diagnostics, Basel, Switzerland, http://www. roche-applied-science.com). Protein yield was determined using the BioRad Protein Assay Dye Reagent Concentrate (BioRad, Hemel Hempstead, U.K., http://www.bio-rad.com). Proteins were loaded equally and separated by SDS-PAGE and transferred to polyvinylidene difluoride membrane (PVDF). Membranes were probed overnight at $4^{\circ} \mathrm{C}$ with antibody against HMGB2 (1:1,000; ab67282, Abcam, Cambridge, U.K., http://www.abcam.com) or for 1 hour at RT with antibody against $\beta$-Actin (1:100,000; A1978, Sigma-Aldrich), followed by incubation with horseradish peroxidase (HRP)-conjugated antirabbit or anti-mouse secondary antibodies (P0448 and P0447, respectively; Dako, Ely, U.K., http://www.dako.com). Chemiluminescent detection was performed using ECL Prime (GE Healthcare), and membranes were imaged on a G:Box Chemi XX6 (Syngene, www.syngene.com/).

\section{Immunohistochemistry, Immunofluorescence, and SA- $\beta$-GAL Staining}

See Supporting Information Methods.

\section{Secretome Analysis}

Conditioned medium was collected from cells transfected with either HMGB2 or NT siRNA, which either remained undifferentiated or were decidualized for 2,4 , or 8 days. Undiluted conditioned medium was assayed for 18 cytokines, chemokines, and growth factors using a multiplex suspension bead immunoassay (Bio-Rad) according to the manufacturer's instructions. Data analysis was carried out using five-parameter logistic regression modeling on the Bio-Plex system [17].

\section{Reverse Transcriptase Quantitative PCR}

Reverse transcriptase quantitative PCR (RT-qPCR) was performed on an Applied Biosystems 7500 system with SDS 1.4 software. For transcript analysis, RNA was extracted from cultured cells using RNA STAT-60 (AMS Biotechnology, Oxford, U.K., www.amsbio.co.uk/), according to the manufacturer's protocol. Purified RNA was solubilized in nuclease-free water and quantitated using the Nanodrop ND-1000 spectrophotometer. For qPCR analysis, $1 \mu \mathrm{g}$ RNA was reverse transcribed using the Quantitect Reverse Transcription Kit (Qiagen, Hilden, Germany, http://www1.qiagen.com), which includes a gDNA removal step, using a combined random hexamer and oligo dT priming strategy. Reactions were diluted fivefold to give an equivalent of $10 \mathrm{ng}$ RNA per $\mu \mathrm{l}$ and stored at $-20^{\circ} \mathrm{C}$. Intronspanning primers were used and primer sequences are tabulated in Supporting Information Table S2. In addition, HMGB2 transcript levels in midluteal endometrial biopsies were measured using nCounter Gene Expression analysis (nanoString Technologies, Seattle, www.nanostring.com/).

\section{Cell Cycle and Proliferation Analyses}

For cell cycle analysis, HESCs in suspension were washed with PBS then fixed with cold $70 \%$ ethanol, treated with RNase A (Qiagen), stained with propidium iodide (Sigma-Aldrich) and subjected to flow cytometry analysis. Cell cycle distribution was assessed using FlowJo software. Real-time adherent cell proliferation was determined by the label-free xCELLigence Real-Time Cell Analyser (RTCA) DP instrument (Roche Diagnostics $\mathrm{GmbH}$ ). Changes in cell index were captured and analyzed using the RTCA Software v1.2.

\section{Statistical Analysis}

Statistical analyses were performed with IBM SPSS version 21 and Graphpad Prism (Graphpad Software, www.graphpad.com/). Distribution normality and equality of variance between groups were examined using the Kolmogorov-Smirnov and Levene's tests, respectively. Where these tests reported significant differences, nonparametric tests were used for downstream comparisons between groups. The histogram presenting distribution of DMRs across the genome was prepared in MATLAB using a $2.5 \mathrm{Mbp}$ window. Sequence logos for over-represented motifs were generated using the Berkeley WebLogo tool [25]. The hypergeometric test for overlap of MeDIP-seq and RNA-seq results was calculated using the expression phyper $(80,7678$, 17691,209,lower.tail=FALSE) in R.

\section{RESULTS}

\section{Differential CpG Methylation in HESCs in RPL Patients}

To investigate the DNA methylome of HESCs, we performed MeDIP-seq, which involves IP of DNA with a 5-methylcytosine antibody followed by deep sequencing, on primary cultures (passage 1) established from four RPL patients and four control subjects (Supporting Information Table S1). Of the 28 million CpGs in the haploid human genome, MeDIP-seq covered approximately $87 \%$ at $100-300$ bp resolution. The overall pattern of methylation, whether at CpG islands, promoters or gene bodies, was similar in both sample sets (Fig. 1A). Analysis of MeDIP-scores, reflecting absolute levels of CpG methylation per $\mathrm{kb}$, identified divergent methylation ( $\geq 2$-fold; $t$ test: $p<.01)$ at 209 loci, of which $48.3 \%$ and $51.7 \%$ were hypomethylated and hypermethylated, respectively, in RPL cultures. The differences were, however, no longer statistically 
significant following Bonferroni correction for multiple comparisons, questioning the pathological relevance of differential methylation in RPL.

To address these concerns, we sequenced the transcriptomes of mid-luteal endometrial biopsies from $10 \mathrm{RPL}$ patients and 10 subfertile women with no history of RPL (Supporting Information Table S1). Principal component analysis segregated the samples into two distinct clusters: a diffuse group (cluster 1) containing $3 \mathrm{RPL}$ and 7 non-RPL samples, and a focused cluster of 10 samples, which included 7 RPL biopsies (cluster 2) (Fig. 1B). This distribution was not accounted for by patient demographics or by the day of the biopsy in the cycle (Supporting Information Table S1) but commensurate with clinical complexity as non-RPL patients formed a heterogeneous group and not all cases of RPL can be attributed to uterine factors. By combining baySeq, DEseq2, and edgeR analyses, we generated a robust list of 3,214 differentially expressed genes (Fig. 1C), depicted with a heatmap of the Z-scores of transcripts per million values in Figure 1D. Gene ontology (GO) analysis showed enrichment of differentially expressed genes in numerous biological categories, including response to stress (GO: 0006950; $p=4.9^{-39}$ ), cell death (GO: 0008219; $p=3.2^{-21}$ ), hemostasis (GO: 0007599; $\left.p=2.1^{-27}\right)$, wound healing (GO: 0042060; $p=2.1^{-24}$ ), immune response (GO: 0006955; $p=5.9^{-28}$ ), and leukocyte migration (GO: 0050900; $p=1.1^{-26}$ ). The latter was validated by immunohistochemistry, which demonstrated higher levels of $\mathrm{CD}^{+} 6^{+}$uterine natural killer cells in cluster 2 samples in keeping with enhanced levels of NCAM1 mRNA (coding neural cell adhesion molecule 1 or CD56) (Supporting Information Fig. S2).

Of the 209 DMRs, 80 (39\%) encompassed putative regulatory regions of endometrial genes deregulated in cluster 2 . This group included several genes that encode for factors relevant to tissue regeneration, for example insulin-like growth factor 1 (IGF1), protection of telomeres protein 1 (POT1), and HMGB2 (Supporting Information Fig. S3). Hypergeometric testing showed a significant overlap between the MeDIP- and RNA-seq datasets $(p=.005)$, suggesting that altered CpG methylation in HESCS may contribute to endometrial dysfunction associated with RPL. It is, however, not possible to draw firm conclusions as multiple cell types besides HESCs, including epithelial, immune and vascular cells, will contribute to altered gene expression in vivo.

\section{Loss of Methylation at CA-Rich Motifs in RPL}

Standard pipelines for MeDIP-seq data a priori assume that methylation is confined to $\mathrm{CpG}$ dinucleotides [26]. We developed a method that removes this assumption from the analysis. This unbiased approach unexpectedly uncovered 2,741 additional DMRs that were hypomethylated $(>1.5$-fold) in three out of four RPL cultures (Fig. 2A, 2B). The DMRs overwhelmingly mapped to CA-rich regions largely devoid of $\mathrm{CPG}$ dinucleotides, many clustering within $15 \mathrm{Mb}$ of the telomeres (Supporting Information Fig. S4). We explored if these DMRs were distinct merely because of CA-richness or whether they harbored additional genomic features. A subset of the data was used to train a motif-based model, which was then applied on the remaining data. Both the motif-based model and the CA-richness criterion achieved very low false positive rates $(\approx 0.004 \%)$, enabling genome-wide application. The motif-based model outperformed the CA-richness criterion indicating that it characterizes DMRs more accurately (Fig. 2C). As shown in Figure 2D, the 10 most significant motifs are characterized by varying periodicities of short $k$-mer repetitiveness, accompanied by a periodicity of the conservation per base pair across these loci. In addition, the statistical scores associated with each locus strongly correlated with the fold-change in methylation between the RPL and control samples $\left(p<10^{-90}\right.$; Fig. 2E). The DMRs were mostly associated with intergenic DNA regions and introns. A survey of publically available chromatin immunoprecipitated-seq data revealed that these CA-rich regions are relatively depleted in transcription factor binding, although there were notable exceptions, such as KAISO (ZBTB33, a component of the $\mathrm{N}$ CoR repression complex [27]) and the orphan nuclear receptor TR4 (NR2C2) (Supporting Information Table S3). GO analysis of nearest genes yielded only a single term (ion channel activity) enriched ( $q$ value $=0.048$ ) in the "Function" category.

Taken together, our unbiased analysis revealed a conspicuous lack of methylation at distinct motifs embedded within CA-rich regions across the HESC genome in RPL cultures. Notably, reanalysis of published MeDIP-seq data indicated that methylation changes in overlapping CA-rich regions are relevant to other pathologies, including transformation of normal Schwann cells into benign and malignant peripheral nerve sheath tumors (Supporting Information Fig. S5) [28]. The magnitude of this differential epigenetic signal is large, more so in tumors than in HESCs, and missed by standard data analysis.

\section{Endometrial Stem Cell Deficiency and RPL}

$\mathrm{CpH}(\mathrm{H}=\mathrm{A} / \mathrm{C} / \mathrm{T})$ methylation is a feature of stem cells, including embryonic stem cells and induced pluripotent stem cells, but lost in most somatic tissues [29-32]. Modification of cytosines at $\mathrm{CpHs}$ is largely dependent on the de novo DNA methyltransferase, DNMT3A [33]. Analysis of independent primary cultures showed that DNMT3A mRNA levels increase in decidualizing cells from control subjects but not RPL patients (Fig. 3A). Matrix-MeDIP-qPCR, that is, microplate-based methylated DNA immunoprecipitation followed by qPCR [16], demonstrated that DNMT3A knockdown in primary HESCs reduces methylation of informative CA-rich but not CpG loci (Fig. 3B, $3 C)$. The loss of an epigenetic signature associated with stemness pointed toward endometrial MSC deficiency in RPL patients. To investigate this further, we used an antibody directed against type 1 integral membrane protein Sushi domain containing 2 (also known as $\mathrm{W} 5 \mathrm{C5}$ ) to isolate perivascular $\left(\mathrm{W}_{5} \mathrm{C}^{+}\right)$and nonperivascular $\left(\mathrm{W} 5 \mathrm{C}^{-}\right)$HESCs from timed mid-luteal biopsies [17, 34]. Analysis of 59 samples showed no difference in the abundance of $\mathrm{W}_{5} \mathrm{C5}^{+}$perivascular cells between RPL and control subjects (Fig. 3D). However, the clonogenicity of $\mathrm{W}_{5} \mathrm{C5}^{+}$and $\mathrm{W}_{5} \mathrm{C}^{-}$cell populations was significantly reduced in RPL $(p=.002$ and $p=.0005$, respectively; Fig. 3E, 3F). Strikingly, no clonogenic $\mathrm{W} 5 \mathrm{C} 5^{-}$cells were recovered from 13 out of 31 (42\%) RPL samples compared to 3 out of $28(11 \%)$ control samples. Furthermore, the abundance of clonogenic $\mathrm{W}_{5} \mathrm{C}^{+}$or $\mathrm{W} 5 \mathrm{C}^{-}$cells correlated negatively with the number of previous miscarriages (Fig. 3G, 3H). Matrix-MeDIP-qPCR analysis revealed a similar inverse correlation between the level of methylation of a randomly selected CA-rich region (chromosome 6) and severity of the RPL phenotype (Fig. 3I). 

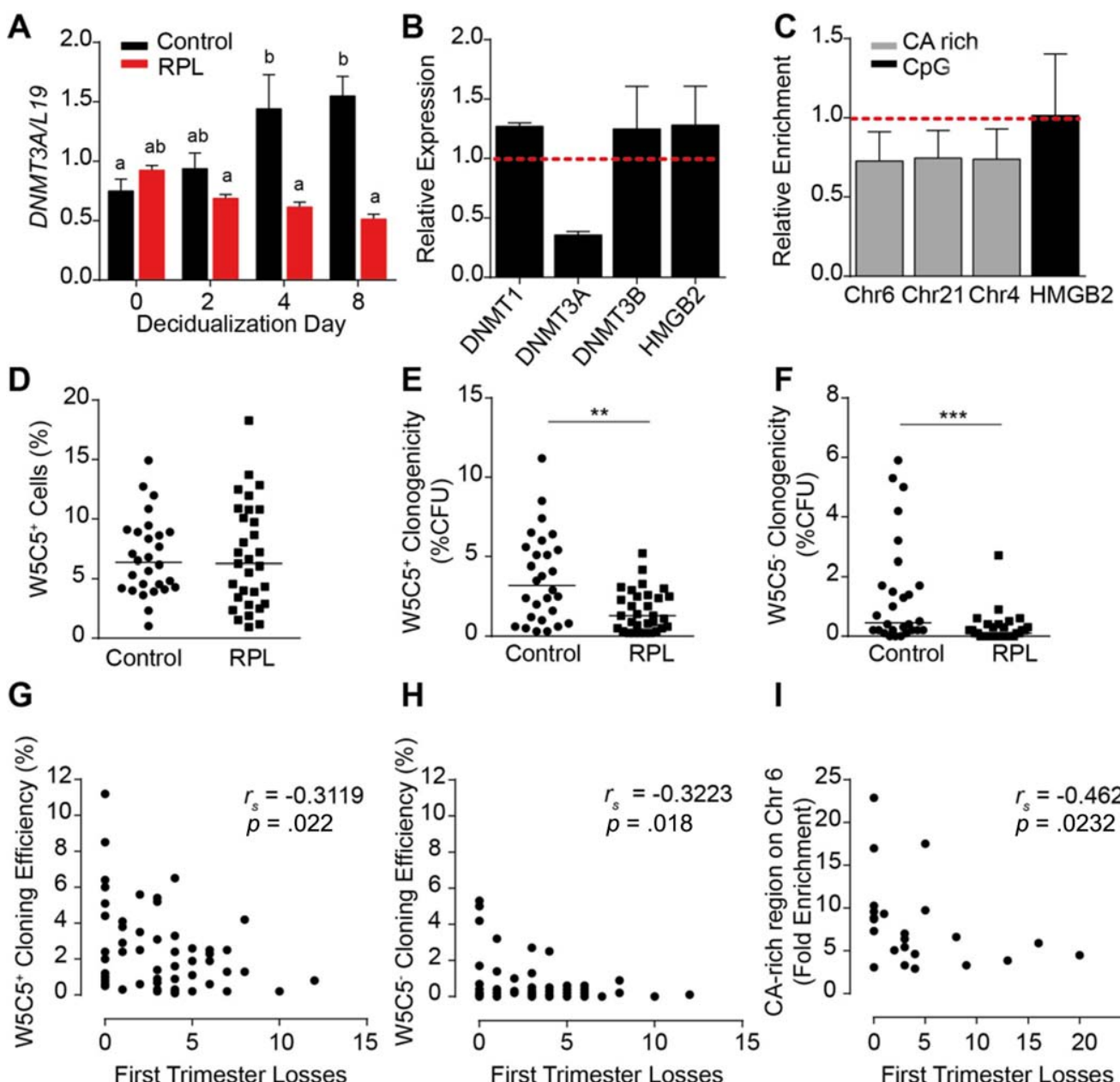

I

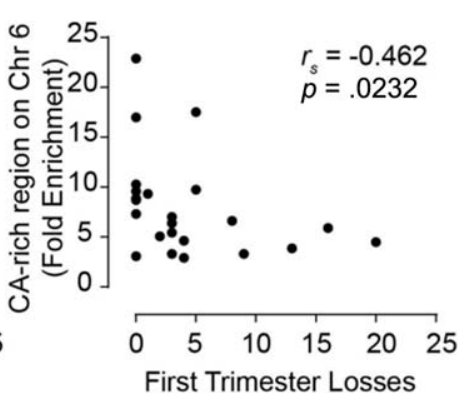

Figure 3. RPL is associated with a deficiency in endometrial clonogenic cell populations. (A): DNMT3A is induced in decidualizing primary human endometrial stromal cell (HESC) cultures from control subjects but not RPL patients. Confluent primary cultures were decidualized using 8-bromo-cAMP and medroxyprogesterone acetate for the indicated time points. Data represent mean \pm SEM of five RPL and five control cultures. Different letters above the error bars indicate that those groups are significantly different from each other at $p<.05$. (B): siRNA-mediated knockdown of DNMT3A did not impact on transcript levels of other DNA methyltransferases or high mobility group protein 2 (HMGB2). The data show the level of expression (mean \pm SEM) upon DNMT3A knockdown relative to transfection of cells with nontargeting siRNA (dotted line) in three HESC cultures. (C): Matrix-MeDIP coupled to quantitative polymerase chain reaction (qPCR) analysis of parallel cultures shows that DNMT3A knockdown modestly but consistently reduces methylation of randomly chosen CA-rich loci on the indicated chromosomes. The level of methylation in HMGB2 gene body was unaltered upon DNMT3A knockdown. (D): Magnetic-activated cell sorting was used to isolate perivascular $\left(\mathrm{W}_{5} \mathrm{C} 5^{+}\right)$and nonperivascular $\left(\mathrm{W}^{2} \mathrm{C}^{-}\right)$endometrial stromal cells from mid-luteal endometrial biopsies. The relative (\%) abundance of $\mathrm{W} 5 \mathrm{C}^{+}$-cells did not differ between biopsies obtained from RPL $(n=34)$ and control subjects $(n=25)(p>.05)$. (E): Colony-forming assays were used to assess clonogenicity, defined by the relative abundance of $\%$ CFU of freshly isolated $\mathrm{W} \mathrm{C} 5^{+}$-cells in RPL and control biopsies. ${ }^{* *}, p=.002$. (F): In parallel, colony-forming assays were performed on freshly isolated $\mathrm{W} 5 \mathrm{C}^{-}$-cells. ${ }^{* * *}, p=.0005$. (G): Spearman's rank was used to assess the relationship between the number of previous pregnancy losses and clonogenicity of $\mathrm{W}_{5} \mathrm{C5}^{+}$-cells (H) and W5C5 ${ }^{-}$-cells. (I): Matrix-MeDIP-qPCR analysis of 24 additional primary HESC cultures shows a negative correlation between the level of methylation at an informative CA-rich region on chromosome 6 and the number of previous pregnancy losses. Abbreviations: CFU, colony forming unit; DNMT, DNA methyltransferase; RPL, recurrent pregnancy loss.

\section{Loss of HMGB2 Impairs Decidual Transformation of HESCS}

We also interrogated putative differentially methylated $\mathrm{CpG}$ loci in the expanded cohort of primary cultures. Intriguingly, methylation levels at CA-rich loci correlated with those in the HMGB2 gene body ( $p=.001$; Fig. $4 \mathrm{~A}$ ). By contrast, no associations were found with methylation of the promoter regions of IGF1 or POT1 (Supporting Information Fig. S6). The impact of DMRs on gene expression is difficult to ascertain, although intragenic methylation tends to enhance transcriptional elongation [23, 35]. Indeed, hypomethylation of HMGB2 gene body was associated with lower expression in cluster 2 samples (Supporting Information Fig. S3). Reduced endometrial HMGB2 expression in RPL patients was further validated in 32 mid-luteal biopsies as well as 10 primary HESC cultures (Supporting Information Fig. S7). Loss of HMGB2 is reportedly a hallmark of replicative senescence in human fibroblasts and associated with inflammation, especially in aging tissues [36-38]. To assess the functional consequences of impaired $H M G B 2$ expression in endometrial cells, primary cultures from control subjects were transfected with either NT or HMGB2 siRNA (Fig. 4B). HMGB2 knockdown greatly impaired the 
A

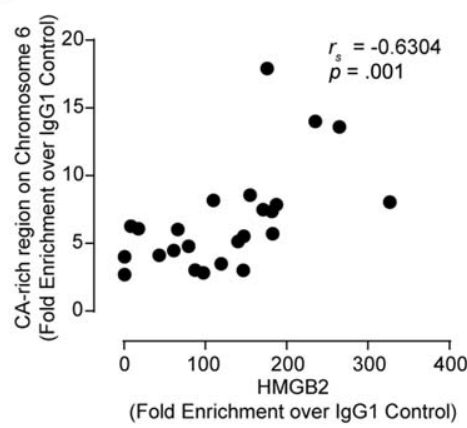

D

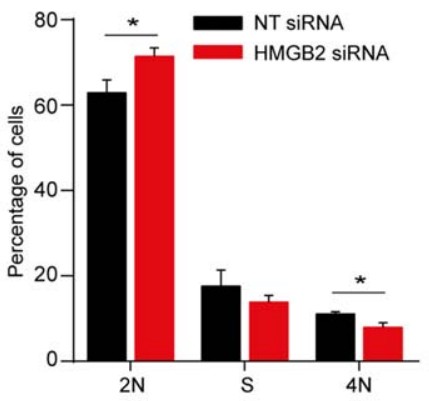

B

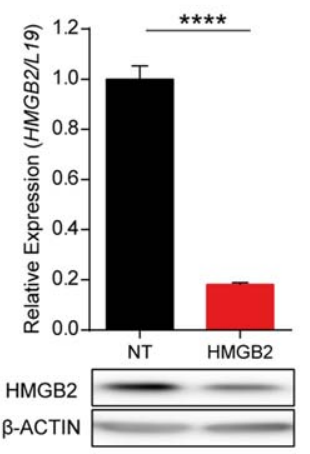

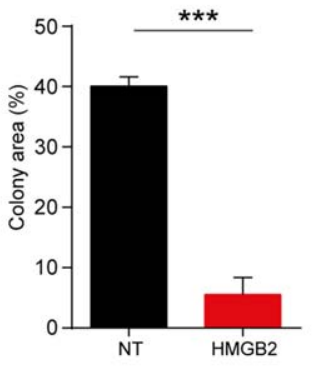

E

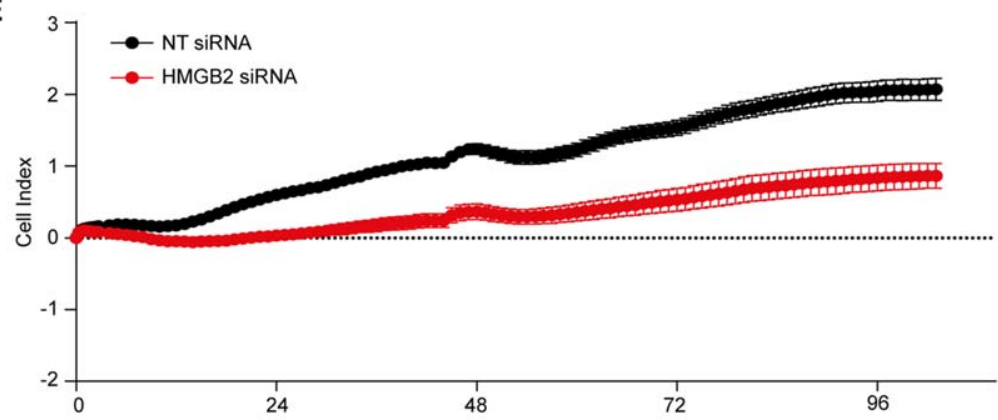

G
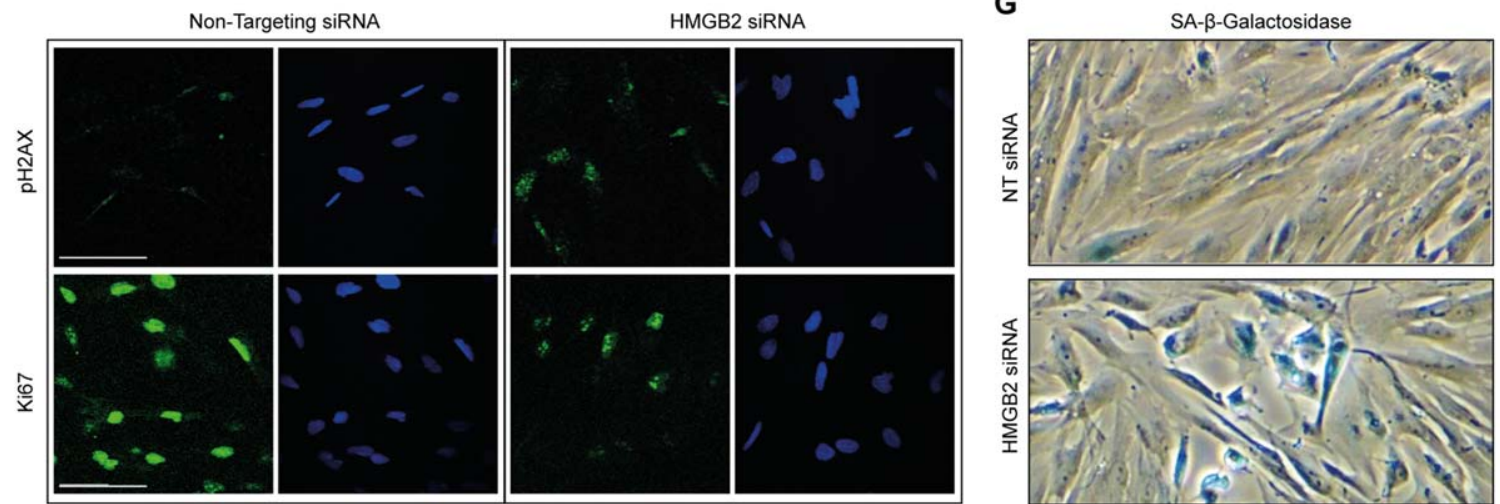

Figure 4. Loss of HMGB2 promotes senescence in human endometrial stromal cells (HESCs). (A): Matrix-MeDIP-qPCR analysis of 24 primary HESC cultures revealed a positive correlation between the level of intragenic HMGB2 methylation and the methylation status of an informative CA-rich region (chromosome 6) $\left(r_{s}=0.6304 ; p=.001\right)$. (B): Knockdown of HMGB2 at mRNA (upper panel) and protein level (lower panel) in response to transfection of primary HESCs with targeted versus NT siRNA. The results show the relative level of HMGB2 knockdown in four primary cultures. Data represent mean $\pm \mathrm{SEM} ; * * *, p<.0001 ., p<.0001$. (C): Colony forming assays of primary cultures first transfected with either NT or HMGB2 siRNA. The right panel shows the total colony area as measured by ImageJ analysis of three independent cultures. The data represent mean colony area ( \pm SEM). ${ }^{* * *}, p<.001$. (D): Flow cytometry analysis of propidium iodide stained primary cultures $(n=3)$ shows that HMGB2 knockdown triggers accumulation of cells at G1/G0 $(2 \mathrm{~N})$ with concomitant reduction in the proportion of cells at $\mathrm{G} 2 / \mathrm{M}(4 \mathrm{~N}) .{ }^{*}, p<.05$. (E): Real-time monitoring of cell growth (xCelligence analysis) for more than 100 hours following transfection with either NT or HMGB2 siRNA. (F): Immunofluorescence staining revealed that HMGB2 knockdown induces loss of antigen $\mathrm{Ki}-67$, a proliferation marker, in parallel with increased expression of the DNA damage mark pH2AX. (G): HMGB2 knockdown increased SA- $\beta$-GAL activity in HESCs when compared to cells transfected with NT siRNA. Scale bar $=50 \mu$ m. Abbreviations: HMGB2, high mobility group protein 2; NT, nontargeting; pH2AX, gamma-H2AX phosphorylation; SA- $\beta$-GAL, senescenceassociated $\beta$-galactosidase.

colony-forming unit-fibroblast activity of primary HESC cultures (Fig. 4C). Flow cytometry analysis of three independent primary cultures revealed accumulation of HESCs in G0/G1 phase of the cycle following transfection with HMGB2 siRNA, which was accompanied by a reduction of cells in $G 2 / M$ phase (Fig. 4D). Real-time monitoring of cell growth more than 100 hours using microelectronic sensor technology (xCEL-
Ligence) confirmed that siRNA-mediated HMGB2 knockdown compromises the proliferative potential of HESCs (Fig. 4E). In parallel with loss of the proliferation antigen Ki-67, knockdown of this chromatin protein also upregulated gamma$\mathrm{H} 2 \mathrm{AX}$ phosphorylation (pH2AX) (Fig. 4F; Supporting Information Fig S8) and induced senescence-associated $\beta$-galactosidase activity (Fig. 4E). Notably, knockdown of HMGB2 was not 
A
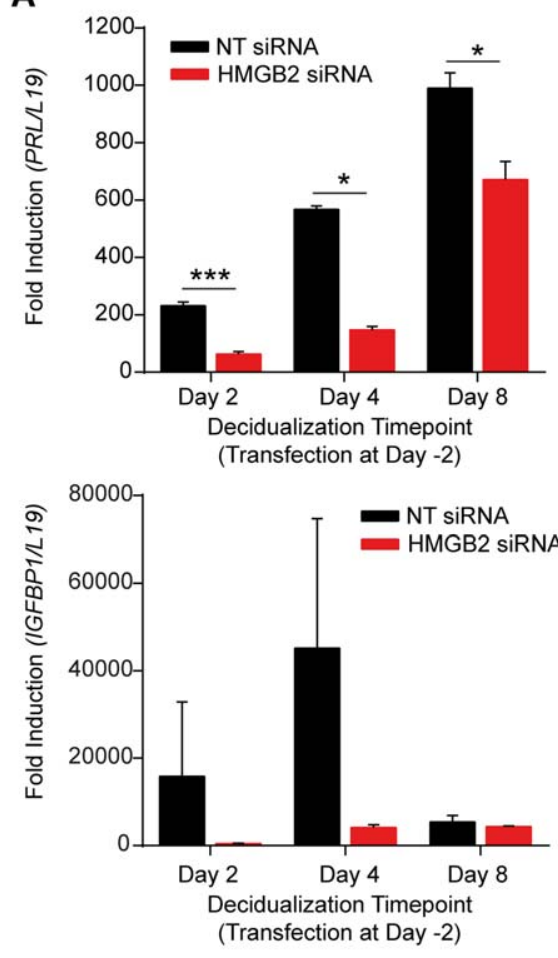

B

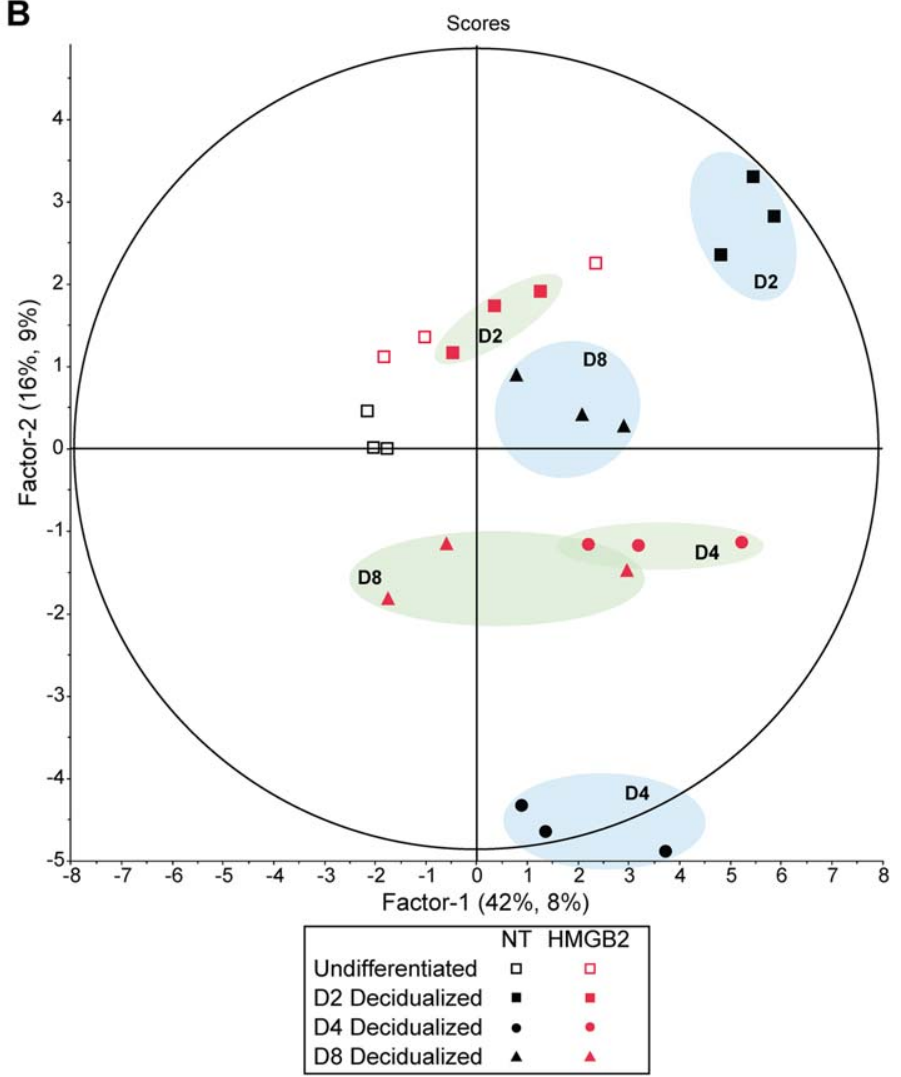

Figure 5. Loss of HMGB2 in HESCs perturbs decidualization. (A): HMGB2 knockdown impairs the induction of $P R L$ and IGFBP1, cardinal decidual marker genes, in primary cultures decidualized for the indicated time points. Data are presented as mean $\pm \mathrm{SEM}, *, p<.05$; $* * *, p<.001$. (B): Two-dimensional partial least squares (PLS) loadings plot of the secretome of undifferentiated cells and cells decidualized 2, 4, or 8 days following transfection with non-targeting (NT) or HMGB2 siRNA. Abbreviations: HMGB2, high mobility group protein 2; NT, nontargeting.

sufficient to reduce methylation of an informative CA-rich locus on chromosome 6 (Supporting Information. S9). Taken together, these observations indicate that a reduction in HMGB2 triggers a DNA-damage response, leading to premature cycle exit and cellular senescence.

We reasoned that accelerated senescence of HESCs would impact on the temporal changes in the decidual secretome that coordinate early implantation events. In agreement, knockdown of HMGB2 was sufficient to compromise induction of $P R L$, the cardinal decidual marker gene, in differentiating HESCs (Fig. 5A). Induction of IGFBP1 varied greatly between primary cultures and was compromised upon HMGB2 knockdown, although not significantly so (Fig. 5A). We expanded our analysis by measuring 18 cytokines and growth factors in the supernatants of three independent primary HESC cultures first transfected either NT or HMGB2 siRNA and then decidualized for 2, 4, or 8 days. Partial least squares regression analysis confirmed that decidualizing HESCs mount a distinct but transient secretory response that is most pronounced between 2 to 4 days of differentiation (Fig. 5B). HMGB2 knockdown had little impact on the secretome of undifferentiated HESCs but blunted the temporal changes in the decidual secretome, as exemplified by secretory profiles of vascular endothelial growth factor (VEGF), beta nerve growth factor (bNGF), fibroblast growth factor 2 (FGF2), and interleukin 8 (IL-8) (Supporting Information Fig. S10). Thus, analysis of the CPG methylome of HESC cultures combined with RNA- sequencing of timed endometrial biopsies suggests that premature senescence and associated tissue inflammation predisposes for RPL.

\section{DiscusSiON}

In response to the postovulatory rise in progesterone levels, differentiating HESCS transit through distinct functional phenotypes, characterized first by an acute proinflammatory phase, which is followed by a profound anti-inflammatory response [11, 15]. The initial inflammatory response renders the endometrium receptive to embryo implantation, whereas acquisition of a mature secretory phenotype enables the endometrium to respond to individual embryos in a manner that either supports further development or facilitates early rejection. RPL is associated with a blunted but prolonged proinflammatory decidual response, which in turn promotes out-of-phase implantation in a hostile environment that predisposes for miscarriage [14, 15]. This pathway is, at least partly, recapitulated in primary HESC cultures from RPL patients and, as shown in this study, is predicated on stem cell deficiency and enhanced senescence.

Unbiased analysis of MeDIP-seq data showed that HESCs from affected women lack a genome-wide epigenetic signature associated with pluripotent cells [29-32]. This signature, defined by DNA methylation at specific motifs in CA-rich loci, is massively amplified in malignant peripheral nerve sheath 
tumors, presumably reflecting the abundance of highly aggressive cancer stem cells $[39,40]$. The function of non-CpG methylation in HESCs requires further investigation. In the adult brain, $\mathrm{CpH}$ methylation has been implicated in gene repression whereas the opposite association was observed in embryonic stem cells $[29,30]$. Recent studies demonstrated that methyl-CpG binding protein 2 (MeCP2) binds to methylated $\mathrm{CpHs}$, albeit with lower affinity than to methylated CpGs [29, 41]. This raises the possibility that dynamic changes in $\mathrm{CpH}$ methylation regulate transcription indirectly by modulating the spatiotemporal binding of MeCP2. Alternatively, methylation could determine access of specific transcription factors, such as the orphan nuclear receptor TR4, to response elements embedded in CA-rich regions (Supporting Information Table S3). The nature of the ensuing transcriptional response, if any, is unclear as most DMRs are located away from promoters in intergenic DNA and introns.

As expected, $\mathrm{CpH}$ but not $\mathrm{CpG}$ methylation in HESCs was dependent on DNMT3A. Intriguingly, DNMT3A transcript levels increased upon decidual transformation of HESC cultures, which suggests that repeated waves of decidualization could play a role in imposing or maintaining endometrial plasticity. Induction of DNMT3A was lacking in decidualizing cultures from RPL patients. Furthermore, analysis of timed endometrial biopsies showed that the level of depletion of endometrial stem cell populations correlates with the severity of RPL phenotype. Lack of epigenetic stemness features in HESCs was further associated with intragenic hypomethylation and reduced expression of $H M G B 2$, coding one of four members of the HMGB family. HMGB2 is a multifaceted protein involved in chromatin bending and DNA damage repair [42, 43]. It also functions as a major progesterone receptor coactivator [44]. Furthermore, HMGB2 is implicated in maintaining stem cell populations in adult tissues and loss of this chromatin protein is a hallmark of replicative senescence of human fibroblasts [36, 45]. In HESCs, knockdown of HMGB2 was sufficient to block clonogenic expansion, impose cell cycle block at G0/G1, activate the DNA damage repair pathway as evidenced by $\mathrm{pH} 2 \mathrm{AX}$, and deregulate decidualization in response to CAMP and progestin signaling. These observations in culture are in line with transcriptome analysis of mid-luteal biopsies, demonstrating that RPL is associated with excessive inflammation, heightened influx of immune cells, cellular stress, and activation of wound healing pathways.

\section{CONCLUSIONS}

As is the case for most adult tissues, resident and bone marrow-derived MSCs are critical for endometrial homeostasis and tissue repair [46-48]. Menstruation, miscarriage, and parturition impose a constant need for recruitment and activation of endometrial progenitor cells. As a consequence of dynamic changes in stem cell populations, the endometrium exhibits extraordinary plasticity, exemplified by its seemingly inexhaustible regenerative capacity and, presumably, its ability to adapt following reproductive failure $[6,11,12]$. Our data indicate that MSC deficiency coupled to premature senescence of HESCs curtails endometrial plasticity. It is tempting to speculate that persistence of this defect for more than several conception cycles accounts for the consecutive nature of miscarriages in RPL patients. This hypothesis requires further testing in longitudinal studies to determine if endometrial assessment in RPL patients is predictive of subsequent pregnancy outcome. If so, harnessing the mechanisms that control endometrial stem cell populations may lead to effective therapies to prevent RPL.

\section{ACKNOWLEDGMENTS}

We thank all the women who participated in this study. We also thank Sean James for excellent technical assistance and to Oleg Denisenko for advice. This work was supported by the Biomedical Research Unit in Reproductive Health and The Genesis Research Trust (P16602 to G.G and J.J.B). This work was also supported by the University Hospitals Coventry and Warwickshire NHS Trust.

\section{AUTHOR CONTRIBUTIONS}

E.S.L., N.P.D., K.M., Y.H.L., Y.-W.C., G.G., J.M., P.J.B, G.P., E.W.F.L, and S.O.: collection and/or assembly of data, data analysis and interpretation, manuscript writing, and final approval of manuscript; J.D.M., J.K.Y.C., and S.T.: administrative support, data analysis and interpretation, manuscript writing, and final approval of manuscript; S.Q.: provision of study material or patients, data analysis and interpretation, manuscript writing, and final approval of manuscript; J.J.B.: conception and design, provision of study material or patients, collection and/ or assembly of data, data analysis and interpretation, manuscript writing, and final approval of manuscript.

\section{Disclosure of POTENTIAL CONFlicts OF INTEREST}

The authors indicate no potential conflicts of interest.

\section{REFERENCES}

1 Evaluation and treatment of recurrent pregnancy loss: A committee opinion. Fertil Steril 2012;98:1103-1111.

2 van den Boogaard E, Kaandorp SP, Franssen MT et al. Consecutive or nonconsecutive recurrent miscarriage: Is there any difference in carrier status? Hum Reprod 2010;25:1411-1414.

3 Stirrat GM. Recurrent miscarriage. Lancet 1990 15;336:673-675.
4 Ogasawara M, Aoki K, Okada S, Suzumori K. Embryonic karyotype of abortuses in relation to the number of previous miscarriages. Fertil Steril 2000;73:300-304.

5 Rai R, Regan L. Recurrent miscarriage. Lancet 2006;368:601-611.

6 Lucas ES, Salker MS, Brosens JJ. Uterine plasticity and reproductive fitness. Reprod Biomed Online 2013;27:506-514.
7 Fragouli E, Alfarawati S, Spath K et al. The origin and impact of embryonic aneuploidy. Hum Genet 2013,132:1001-113

8 Vanneste $\mathrm{E}$, Voet $\mathrm{T}$, Le Caignec $\mathrm{C}$ et al. Chromosome instability is common in human cleavage-stage embryos. Nat Med 2009;15: 577-583.

9 Brosens JJ, Salker MS, Teklenburg G et al. Uterine selection of human embryos at implantation. Sci Rep 2014;4:3894. 
10 Teklenburg G, Salker M, Molokhia M et al. Natural selection of human embryos: Decidualizing endometrial stromal cells serve as sensors of embryo quality upon implantation. PLoS One 2010;5:e10258.

11 Gellersen B, Brosens JJ. Cyclic decidualization of the human endometrium in reproductive health and failure. Endocr Rev 2014; 35:851-905.

12 Macklon NS, Brosens JJ. The human endometrium as a sensor of embryo quality. Biol Reprod 2014;91:98.

13 Salker M, Teklenburg G, Molokhia M et al. Natural selection of human embryos: Impaired decidualization of the endometrium disables embryo-maternal interactieons and causes recurrent pregnant loss. PLoS One 2010;5:e10287.

14 Salker MS, Christian M, Steel JH et al. Deregulation of the serum- and glucocorticoid-inducible kinase SGK1 in the endometrium causes reproductive failure. Nat Med 2011;17:1509-1513.

15 Salker MS, Nautiyal J, Steel JH et al. Disordered IL-33/ST2 activation in decidualizing stromal cells prolongs uterine receptivity in women with recurrent pregnancy loss. PLoS One 2012;7:e52252.

$16 \mathrm{Yu}$ J, Feng $\mathrm{Q}$, Ruan $\mathrm{Y}$ et al.Microplatebased platform for combined chromatin and DNA methylation immunoprecipitation assays. BMC Mol Biol 2011;12:49.

17 Murakami K, Lee YH, Lucas ES et al Decidualization induces a secretome switch in perivascular niche cells of the human endometrium. Endocrinology. 2014;155: 4542-4553.

18 Wagner GP, Kin K, Lynch VJ. Measurement of mRNA abundance using RNA-seq data: RPKM measure is inconsistent among samples. Theory Biosci 2012;131:281-285.

19 Hardcastle TJ, Kelly KA. baySeq: Empirical Bayesian methods for identifying differential expression in sequence count data. BMC Bioinform 2010;11:422.

20 Love MI, Huber W, Anders S. Moderated estimation of fold change and dispersion for RNA-seq data with DESeq2. Genome Biol 2014;15:550.

21 Robinson MD, McCarthy DJ, Smyth GK edgeR: a Bioconductor package for differential expression analysis of digital gene expres sion data. Bioinformatics 2010;26:139-140.

22 Robinson MD, Oshlack A. A scaling normalization method for differential expression analysis of RNA-seq data. Genome Biol 2010; 11:R25.

23 Maunakea AK, Nagarajan RP, Bilenky M et al. Conserved role of intragenic DNA methylation in regulating alternative promoters. Nature 2010;466:253-257.
24 Jiang $H$, Wang $F$, Dyer NP et al. CisGenome Browser: A flexible tool for genomic data visualization. Bioinformatics 2010;26 1781-1782.

25 Crooks GE, Hon G, Chandonia JM et al. WebLogo: A sequence logo generator. Genome Res 2004;14:1188-1190.

26 Down TA, Rakyan VK, Turner DJ et al. A Bayesian deconvolution strategy for immunoprecipitation-based DNA methylome analysis. Nat Biotechnol 2008;26:779-785. 27 Yoon HG, Chan DW, Reynolds $A B$ et al. $\mathrm{N}$-CoR mediates DNA methylation-dependent repression through a methyl CPG binding protein Kaiso. Mol Cell 2003;12:723-734.

28 Feber A, Wilson GA, Zhang $L$ et al. Comparative methylome analysis of benign and malignant peripheral nerve sheath tumors. Genome Res 2011;21:515-524.

29 Guo JU, Su Y, Shin JH et al. Distribution, recognition and regulation of non-CpG methylation in the adult mammalian brain. Nat Neurosci 2014;17:215-222.

30 Lister R, Pelizzola M, Kida YS et al. Hotspots of aberrant epigenomic reprogramming in human induced pluripotent stem cells. Nature 2011;471:68-73.

31 Ramsahoye $\mathrm{BH}$, Biniszkiewicz D, Lyko $\mathrm{F}$ et al. Non-CpG methylation is prevalent in embryonic stem cells and may be mediated by DNA methyltransferase 3a. Proc Natl Acad Sci USA 2000;97:5237-5242.

32 Ziller MJ, Muller F, Liao J, Zhang Y, Gu $\mathrm{H}$, Bock $\mathrm{C}$, et al. Genomic distribution and inter-sample variation of non-CpG methylation across human cell types. PLoS Genet 2011;7:e1002389.

33 Yokochi T, Robertson KD. Preferentia methylation of unmethylated DNA by mammalian de novo DNA methyltransferase Dnmt3a. J Biol Chem 2002;277:11735-11745. 34 Masuda H, Anwar SS, Buhring $\mathrm{HJ}$ et al. A novel marker of human endometrial mesenchymal stem-like cells. Cell Transplant 2012;21:2201-2214.

35 Lorincz MC, Dickerson DR, Schmitt $M$ et al. Intragenic DNA methylation alters chromatin structure and elongation efficiency in mammalian cells. Nat Struct Mol Biol 2004; 11:1068-1075.

36 Biniossek ML, Lechel A, Rudolph KL et al. Quantitative proteomic profiling of tumor cell response to telomere dysfunction using isotope-coded protein labeling (ICPL) reveals interaction network of candidate senescence markers. J Proteomics 2013 Oct 8;91:515-35.

37 Taniguchi N, Carames B, Kawakami Y et al. Chromatin protein HMGB2 regulates articular cartilage surface maintenance via beta-catenin pathway. Proc Natl Acad Sci USA 2009;106:16817-16822.

38 Taniguchi N, Carames B, Ronfani L et al. Aging-related loss of the chromatin protein HMGB2 in articular cartilage is linked to reduced cellularity and osteoarthritis. Proc Natl Acad Sci USA 2009;106:1181-1186.

39 Spyra M, Kluwe L, Hagel C et al. Cancer stem cell-like cells derived from malignant peripheral nerve sheath tumors. PLoS One 2011;6:e21099.

40 Li Y, Rogoff HA, Keates $S$ et al. Suppression of cancer relapse and metastasis by inhibiting cancer stemness. Proc Natl Acad Sci USA 2015;112:1839-1844.

41 Chen L, Chen K, Lavery LA et al. MeCP2 binds to non-CG methylated DNA as neurons mature, influencing transcription and the timing of onset for Rett syndrome. Proc Natl Acad Sci USA 2015;112:5509-5514.

42 Agresti A, Bianchi ME. HMGB proteins and gene expression. Curr Opin Genet Dev 2003;13:170-178.

43 Shin YJ, Kim MS, Lee J et al. High-mobility group box 2 (HMGB2) modulates radioresponse and is downregulated by p53 in colorectal cancer cell. Cancer Biol Ther 2013; 14:213-221.

44 Boonyaratanakornkit V, Melvin V, Prendergast $P$ et al. High-mobility group chromatin proteins 1 and 2 functionally interact with steroid hormone receptors to enhance their DNA binding in vitro and transcriptional activity in mammalian cells. Mol Cell Biol 1998;18:4471-4487.

45 Taniguchi N, Carames B, Hsu E et al. Expression patterns and function of chromatin protein HMGB2 during mesenchymal stem cell differentiation. J Biol Chem 2011; 286:41489-41498.

46 Alawadhi F, Du H, Cakmak H et al. Bone marrow-derived stem cell (BMDSC) transplantation improves fertility in a murine model of Asherman's syndrome. PLoS One 2014;9: e96662.

47 Cao M, Chan RW, Yeung WS. Labelretaining stromal cells in mouse endometrium awaken for expansion and repair after parturition. Stem Cells Dev 2015; 24:768780.

48 Gargett CE, Nguyen HP, Ye L. Endometrial regeneration and endometrial stem/progenitor cells. Rev Endocr Metab Disord 2012; 13:235-251.

49 Quenby S, Nik H, Innes B et al. Uterine natural killer cells and angiogenesis in recurrent reproductive failure. Hum Reprod 2009; 24:45-54. 